\title{
THE HUREWICZ HOMOMORPHISM AND NUMERICAL FORMS
}

\author{
J. R. HUBBUCK \\ Department of Mathematical Sciences, University of Aberdeen \\ Aberdeen, Scotland, AB24 3UE, UK \\ E-mail: j.hubbuck@maths.abdn.ac.uk
}

1. Introduction. In introducing his series of lectures at the conference, Hans Baues discussed the question of classifying the homotopy types of indecomposable stable finite complexes, mentioning logical problems which might arise in such a programme. The data he chose to use in the classification of small complexes, stressed the role of the classical Hurewicz homomorphism from homotopy to homology groups. This short note addresses both aspects, but with a different approach. In the first section, we prove a theorem on the Hurewicz homomorphism for a product of complex projective spaces in the stable category. In the second section, we discuss its relevance to stable decompositions of some spaces associated with the unitary group. The mathematics is joint work with Michael Crabb.

Let $P$ denote a product of $n$-copies of $P^{\infty}$, infinite dimensional complex projective space. When discussing the unitary group, it will be more natural to think of $P$ as $B T$, with $T$ a maximal torus in $U(n)$. As $P$ is an Eilenberg-Maclane space, its homotopy groups are clear. This latter is not true if we consider its suspensions $\Sigma^{t} P$. Let

$$
h_{r}^{k}: \pi_{r}(P) \rightarrow H_{r}(P, \mathbb{Z} / k \mathbb{Z})
$$

be the stable Hurewicz homomorphism, or

$$
h_{r}^{k}: \pi_{r+t}\left(\Sigma^{t} P\right) \rightarrow H_{r+t}\left(\Sigma^{t} P, \mathbb{Z} / k \mathbb{Z}\right)
$$

for any positive $t$, where $k$ is a fixed positive integer and the coefficients in the homology group arise by tensoring the integral homology with $\mathbb{Z} / k \mathbb{Z}$.

THEOREM 1.1. There exists an integer $K(n, k)$ such that $h_{r}^{k}$ is the zero homomorphism whenever $r>2 K(n, k)$.

1991 Mathematics Subject Classification: Primary 55Q99; Secondary 11E76.

The paper is in final form and no version of it will be published elsewhere. 
At present, the theorem is primarily a qualitative result as the currently known upper bounds on the integers $K(n, k)$ when $n>2$ are too large to be useful for computation. Further progress on determining the indecomposable summands of the spaces discussed in the second section using these techniques depends upon obtaining better results on the $K(n, k)$

We describe the number theory needed for the proof of Theorem 1.1 from [7].

A rational polynomial $p(\xi) \in \mathbf{Q}[\xi]$ is a numerical polynomial, where we write $\xi=$ $\left(\xi_{1}, \xi_{2}, \ldots, \xi_{n}\right)$, if $p(m) \in \mathbb{Z}$ whenever $m \in \mathbb{Z}^{n}$. A basis for numerical polynomials over $\mathbb{Z}$ is

$$
\left.\left\{\gamma_{i_{1}}\left(\xi_{1}\right) \gamma_{i_{2}}\left(\xi_{2}\right) \ldots \gamma_{i_{n}}\left(\xi_{n}\right)\right)\right\}, \quad i_{j} \geq 0
$$

where $\gamma_{i}(\xi)$ is the binomial polynomial $\xi .(\xi-1) \ldots(\xi-i+1) / i$ !.

A numerical polynomial is a numerical $M$-form if it is homogeneous of degree $M$. An example of an $n(n+1) / 2$-form is given by

$$
\operatorname{det}\left\{\gamma_{i}\left(\xi_{j}\right)\right\}, \quad 1 \leq i, j \leq n .
$$

Any numerical $M$-form in $n$-variables can be written as

$$
p(\xi)=\sum a_{i_{1}, i_{2}, \ldots, i_{n}} \frac{\xi_{1}^{i_{1}}}{i_{1} !} \cdot \frac{\xi_{2}^{i_{2}}}{i_{2} !} \ldots \frac{\xi_{n}^{i_{n}}}{i_{n} !} .
$$

where $\sum i_{j}=M$ and each $a_{i_{1}, i_{2}, \ldots, i_{n}} \in \mathbb{Z}$. We write $A(p(\xi))$ for the highest common factor of the coefficients $a_{i_{1}, i_{2}, \ldots, i_{n}}$; this is an invariant of $p(\xi)$ under linear change of variables over $\mathbb{Z}$.

Corollary 1.4 of [7] gives the following.

THEOREM 1.2. Let $k$ be a fixed positive integer. There exists a smallest positive integer $K(n, k)$ such that whenever $p(\xi)$ is a numerical $M$-form in n-variables and $M>K(n, k)$, then $k$ divides $A(p(\xi))$.

There are completely analogous results when $\mathbb{Z}$ is replaced by $\mathbb{Z}_{(p)}$, the integers localised at a prime $p$.

We deduce Theorem 1.1 from Theorem 1.2 using ordinary complex $K$-homology theory and dual Adams operators. (It is convenient to use $p$-local connective $K$-theory and stable Adams operators when working at a prime $p$.) In complex $K$-homology theory, $K_{0}\left(P^{\infty}\right)$ is a filtered ring using the CW-filtration and the Pontrjagin product. If $\xi \in K_{0}\left(P^{\infty}\right)$ is "dual" to the reduced Hopf line bundle in $K$-cohomology, a basis for $K_{0}\left(P^{\infty}\right)$ is $\left\{\gamma_{i}(\xi)\right\}, i \geq 0$. Let $\xi_{i}$ denote

$$
1 \otimes 1 \otimes \ldots 1 \otimes \xi \otimes 1 \otimes \ldots \otimes 1
$$

with $\xi$ in the $i$-th position. The Künneth formula then enables us to identify $K_{0}(P)$ with the linear space of numerical polynomials. The dual Adams operator $\psi_{\alpha}$ satisfies $\psi_{\alpha}\left(\xi_{i}\right)=\alpha \xi_{i}$. So the eigenspace corresponding to $\alpha^{M}$ is precisely the space of numerical $M$-forms.

The associated graded ring of $K_{0}(P)$ is $\bigoplus_{i>0} H_{2 i}(P, \mathbb{Z})$ ). If the image of $\xi_{i}$ is $x_{i}$ under the quotient map to $\left.K_{0}(P)_{2} / K_{0}(P)_{1} \equiv H_{2}(P, \mathbb{Z})\right)$, then the image of any $M$-form 
$p(\xi) \in K_{0}(P)_{2 M}$ is $p(x)$ in $H_{2 M}(P, \mathbb{Z})$, where $x=\left(x_{1}, x_{2}, \ldots x_{n}\right)$. A basis in $H_{*}(P, \mathbb{Z})$ is

where $i_{j} \geq 0$.

$$
\left\{\frac{x_{1}^{i_{1}}}{i_{1} !} \cdot \frac{x_{2}^{i_{2}}}{i_{2} !} \ldots \frac{x_{n}^{i_{n}}}{i_{n} !}\right\}
$$

Let $f: S^{2 q} \rightarrow P$ be a stable map realized as $f: S^{2 q+2 u} \rightarrow \Sigma^{2 u} P$. When $w \in$ $\tilde{K}\left(S^{2 q+2 u}\right)$ is a generator, $\psi_{\alpha}(w)=\alpha^{q+u} w$ and so $\psi_{\alpha}\left(f_{*} w\right)=\alpha^{q+u} f_{*} w$. If $v \in \tilde{K}_{0}(P)$ suspends to $f_{*} w, \psi_{\alpha}(v)=\alpha^{q} v \in \tilde{K}_{0}(P)_{2 q}$ and so, as above, $v$ is a numerical $q$-form $p(\xi) \in K_{0}(P)$. If $\bar{w} \in H_{2 q+2 u}\left(S^{2 q+2 u}\right)$ is the generator corresponding to $w, f_{*}(\bar{w})=p(x)$, by naturality.

So, in the language of stable homotopy, we have proved the following lemma.

Lemma 1.3. Let $f: S^{2 q} \rightarrow P$ be a stable map, $u \in \tilde{K}_{0}\left(S^{2 q}\right)$ a generator with corresponding generator $\bar{u} \in H_{2 q}\left(S^{2 q}, \mathbb{Z}\right)$. Then $f_{*}(u)$ is a numerical $q$-form $p(\xi) \in K_{0}(P)$ and $f_{*}(\bar{u})=p(x) \in H_{2 q}(P, \mathbb{Z})$.

Theorem 1.1 follows from Lemma 1.3 and Theorem 1.2. Let $[f] \in \pi_{r+t}\left(\Sigma^{t} P\right)$. Theorem 1.1 has no content unless $r$ and $t$ have the same parity and as the conclusion is preserved under suspensions (but not de-suspensions), we can assume that both are even integers. So we have a stable map $f: S^{2 q} \rightarrow P$ where $2 q=r$ and $f_{*}(u)=p(\xi) \in K_{0}(P)$ is a numerical $q$-form. So by Theorem $1.2, k$ divides $A(p(x))$ and $p(x)=0$ in $H_{2 q}(P, \mathbb{Z} / k \mathbb{Z})$ or in $H_{r+t}\left(\Sigma^{t} P, \mathbb{Z} / k \mathbb{Z}\right)$ when $q>K(n, k)$. Thus $h_{r}^{k}([f])=0$ if $r>2 K(n, k)$.

2. Applications. Theorem 1.2 was proved to help in the investigation of the stable homotopy decomposability of spaces associated with connected Lie groups. A space or spectrum $X$ is indecomposable if it cannot be expressed as a wedge of two homotopically non-trivial spaces or spectra. The basic strategy is routine. If $X$ is a complex of finite type and $X \simeq Y \vee Z$ where $Y$ is $2 q-1$ connected and $H_{2 q}(Y, \mathbb{Z})$ is infinite, then there exists a stable map $f: S^{2 q} \rightarrow Y$ inducing an injection in homology in degree $2 q$ by inclusion of a bottom cell. In what follows, we discuss the significance of Theorem 1.1 for $B U(n), U(n)$ and $\Omega S U(n)$; near complete results are known only for $n=1$ or $2[3]$, using number theory established in [1].

The idea can be illustrated for $B G$ with $G$ a compact, connected Lie group of rank $n$ and $T$ a maximal torus. The infinite space $B G$ is rich in cohomology and if one can show that there are few potential summands and estimate their connectivities, a study of cohomology operations can lead to a complete determination of these summands. So we suppose that $B G$ has a stable summand $Y$ as in the paragraph above. We consider the stable composition

$$
S^{2 q} \rightarrow Y \rightarrow B G_{+} \stackrel{i^{!}}{\rightarrow} B T_{+} \stackrel{i}{\rightarrow} B G_{+}
$$

where the first two maps and last map $i$ are inclusions and the penultimate map $i^{!}$is a Becker-Gottlieb transfer. The composition $\left(i . i^{!}\right)_{*}: H_{*}(B G, \mathbb{Z}) \rightarrow H_{*}(B G, \mathbb{Z})$ is multiplication by $\chi(G / T)$. So if $\chi(G / T)$ does not divide $k$ and $f: S^{2 q} \rightarrow B T_{+}$is part of the composition above, $h_{2 q}^{k}[f]$ is non-zero and by Theorem 1.1, $q \leq K(n, k)$. Thus for each summand in a decomposition of $B G$, there is a numerical form $p(x)$ of degree less than or equal to $K(n, k)$ which does not vanish in $H_{*}(B G, \mathbb{Z} / k \mathbb{Z})$ by Lemma 1.3 . 
There are well known stable decompositions of $B U(n), U(n)$ and $\Omega S U(n)$. A good reference is [2], except that the splitting for $\Omega S U(n)$, established by Mitchell and Richter, was not known at the time and remains unpublished; a later paper [5] generalizing the results contains the details needed to construct a proof.

The space $B U(n)$ is filtered by inclusions

$$
* \subset B U(1)=P^{\infty} \subset U(2) \subset \ldots \subset U(n)
$$

and $M U(i) \simeq B U(i) / B U(i-1)$. Snaith [10] proved that

$$
B U(n) \simeq \bigvee_{1 \leq i \leq n} M U(i)
$$

and so it is the indecomposability of the $M U(i)$ which is of interest.

The homology group $H_{*}\left(P^{\infty}, \mathbb{Z}\right)$ has a basis $\left\{b_{i}\right\}, i \geq 0$, where the degree of $b_{i}$ is $2 i$ and $b_{1}$ is dual to the Euler class. Under the inclusion of $P^{\infty}$ in $B U(n), H_{*}(B U(n), \mathbb{Z}) \subset$ $H_{*}(B U, \mathbb{Z})=\mathbb{Z}\left[b_{i}\right], i \geq 0$, is spanned by all monomials of homogeneous degree $\leq n$. So $\tilde{H}_{*}(M U(n), \mathbb{Z})$ can be identified with the subspace spanned by monomials of degree $n$.

In applying Theorem 1.1 and Lemma 1.3, the most efficient results occur when $k=2^{\beta}$. As $\chi(U(n) / T)=n$ !, we set $\beta=\nu_{2}(n !)$ in the discussion above, and deduce that if $Y$ is a non-trivial stable summand of $M U(n)$, its connectivity is not greater than $2 K\left(n, 2^{\beta}\right)$. In particular when $n=2,2 K(2,4)=24$. Then using Lemma 1.3, one seeks to find numerical $M$-forms of degree $\leq 12$ which give non-zero classes in $H_{*}(B T, \mathbb{Z} / 4 \mathbb{Z})$. If one also uses the fact that the map $i^{!}: H_{*}(B U(2), \mathbb{Z}) \rightarrow H_{*}(B T, \mathbb{Z})$ is a symmetrization map, a short computation shows that there are only two possible elements in $\tilde{H}_{*}(M U(2), \mathbb{Z} / 4 \mathbb{Z})$ which could represent the lowest degree classes of summands; these are $b_{1}^{2}$ and $b_{1} b_{3}+2 b_{2}^{2}$. We deduce that $M U(2)$ has at most two stable summands. The technical details will appear in [4], where it is shown that this is the best possible result in the sense that MU(2) splits into a wedge of two spectra provided one inverts the prime 3 .

There are also stable splittings of $U(n)$ and $\Omega S U(n)$. Let $L \in P\left(\mathbb{C}^{n}\right), \pi_{L}: \mathbb{C}^{n} \rightarrow \mathbb{C}^{n}$ be orthogonal projection onto $L$ and $\lambda$ be a complex number of modulus 1 . Hopf defines the pseudo-reflection $\rho_{L}(\lambda) \in U(n)$ by setting $\rho_{L}(\lambda)=\lambda \pi_{L}+\left(I-\pi_{L}\right)$. So taking $\Omega U(n)=\operatorname{Map}_{*}\left\{S^{1}, U(n)\right\}$, we have $\rho_{L} \in \Omega U(n)$. We can define subspaces of $\Omega U(n)$ by setting

$$
\Omega^{(k)} U(n)=\left\{f \in \Omega U(n): f=\rho_{L_{1}} \cdot \rho_{L_{2}} \ldots \rho_{L_{k}}\right\}
$$

where the product is induced from that of $U(n)$ and $L_{i} \in P\left(\mathbb{C}^{n}\right)$. These lie in different components, but if we choose a $U(1) \subset U(n)$ and use the projection $U(n) \rightarrow U(n) / U(1)=$ $S U(n)$, we obtain a Mitchell filtration of $\Omega S U(n)$,

$$
* \subset \Omega^{(1)} S U(n)=P\left(\mathbb{C}^{n}\right) \subset \Omega^{(2)} S U(n) \subset \ldots \subset \Omega^{(i)} S U(n) \subset \ldots \subset \Omega S U(n) .
$$

Let $W_{k}(n)=\Omega^{(k)} S U(n) / \Omega^{(k-1)} S U(n)$. The theorem of Mitchell and Richter states that

$$
\Omega S U(n) \simeq \bigvee_{k \geq 1} W_{k}(n)
$$

The spaces $W_{k}(n)$ have the homotopy types of finite complexes. 
The Pontrjagin ring $H_{*}(S U(n), \mathbb{Z})=\mathbb{Z}\left[b_{i}\right] \equiv S^{*}\left\{b_{i}\right\}$, where $1 \leq i \leq n-1$, as $\Omega S U \simeq$ $B U$ and $H_{*}(\Omega S U(n), \mathbb{Z}) \subset H_{*}(\Omega S U, \mathbb{Z})$. Then $H_{*}\left(\Omega^{(k)} S U(n), \mathbb{Z}\right)=\bigoplus_{h \leq k} S^{h}\left\{b_{i}\right\}$ and so $H_{*}\left(W_{k}(n), \mathbb{Z}\right)=S^{k}\left\{b_{i}\right\}$, the linear space spanned by monomials of degree $k$ in $b_{i}$, $1 \leq i \leq n-1$. In particular $W_{k}(\infty) \simeq M U(k)$. The earlier discussion relating to $M U(k)$ can therefore be applied to $W_{k}(n) \subset M U(k)$. In particular when $k=2$, we deduce that $W_{2}(n)$ can have at most two stable summands by the same argument as for $M U(2)$. But whereas $M U(2)$ splits at the prime $2, W_{2}(n)$ is indecomposable; a routine though delicate computation shows that one of the homology classes $b_{n-1}^{2}$ or $b_{n-2} b_{n-1}$, depending upon the parity of $n$, cannot lie in either potential summand. Summarising the result for $n=2$, we have the following result.

TheOREM 2.1. (a) $W_{2}(n)$ is stably indecomposable (at the prime 2 ).

(b) $W_{2}(\infty)=M U(2)$ splits as a wedge of two indecomposable spectra, if the prime 3 is inverted.

The technical details needed for the proof and related results will appear in [4].

The pseudo-reflection $\rho_{L}(\lambda) \in U(n)$ also leads to a filtration on $U(n)$. Let

$$
U(n)^{(k)}=\left\{\rho_{L_{1}}\left(\lambda_{1}\right) \cdot \rho_{L_{2}}\left(\lambda_{2}\right) \ldots \rho_{L_{k}}\left(\lambda_{k}\right): L_{i} \in P\left(\mathbb{C}^{n}\right)\right\}
$$

where $\lambda_{i} \in \mathbb{C}$ with modulus 1 . Then

$$
* \subset U(n)^{(1)}=\Sigma\left(P\left(\mathbb{C}^{n}\right)^{+}\right) \subset U(n)^{(2)} \subset \ldots \subset U(n)^{(n)}=U(n) .
$$

We set $X_{k}(n)=U(n)^{(k)} / U(n)^{(k-1)}$. There is an induced filtration on $S U(n)$ and we define $S X_{k}(n)=S U(n)^{(k)} / S U(n)^{(k-1)}$. A part of the main theorem of [9] gives the following stable decompositions.

$$
\begin{gathered}
U(n) \simeq \bigvee_{1 \leq k \leq n} X_{k}(n) \\
S U(n) \simeq \bigvee_{1 \leq k \leq n-1} S X_{k}(n)
\end{gathered}
$$

As $U(n)$ is homeomorphic to $S^{1} \times S U(n)$, stably $X_{k}(n) \simeq \Sigma S X_{k-1}(n) \vee S X_{k}(n)$, and so we are interested in the indecomposability of the stable finite complexes $S X_{k}(n)$.

At the level of homology $H_{*}(S U(n), \mathbb{Z}) \equiv \bigwedge^{*}\left\{\Sigma b_{i}\right\}, 1 \leq i \leq n-1$ and $H_{*}\left(S U(n)^{(k)}, \mathbb{Z}\right)$ $\equiv \bigoplus_{j \leq k} \Lambda^{k}\left\{\Sigma b_{i}\right\}$. So $H_{*}\left(W_{k}(n), \mathbb{Z}\right)$ can be identified with $\bigwedge^{k}\left\{\Sigma b_{i}\right\}, 1 \leq i \leq n-1$, the space spanned by $\left\{\Sigma b_{i_{1}} \wedge \Sigma b_{i_{2}} \wedge \ldots \wedge \Sigma b_{i_{k}}\right\}, i_{1}<i_{2}<\ldots<i_{k}$.

Let $G$ be a compact Lie group with Lie algebra $g$ and denote by $\lambda(G)$ the Lie algebra bundle $E G \times_{G} g$ over $B G$. The transfer construction applied to the bundle

$$
B T=E U(k) / T \rightarrow B U(k)=E U(k) / U(k)
$$

defines a map of Thom spectra $i^{!}: B U(n)^{\lambda(U(n))} \rightarrow B T^{\lambda(T)}$. But $B T^{\lambda(T)}=\Sigma^{k}\left(B T_{+}\right)$ and $B U(k)^{\lambda(U(k))}=X_{k}(\infty)$.

It is proved in Lemma 4.1 of [3] that in homology

$$
i_{*}^{!}: \tilde{H}_{*}\left(X_{k}(\infty), \mathbb{Z}\right) \rightarrow \tilde{H}_{*}\left(\Sigma^{k} B T_{+}, \mathbb{Z}\right)
$$

is the skew-symmetrisation map and as such is a monomorphism in homology with $\mathbb{Z} / 2 \mathbb{Z}$ coefficients. Using a line of reasoning similar to that above, we deduce that if $Y$ is a nontrivial summand of $S X_{k}(\infty)$ or of $S X_{k}(n)$, then it is at most $k+2 K(n, 2)-1$ connected. When $k=2$, there are only 2 classes in $\tilde{H}_{*}\left(S X_{2}(\infty), \mathbb{Z} / 2 \mathbb{Z}\right)$ which can represent the 
lowest dimensional classes of stable summands, $\Sigma b_{1} \wedge \Sigma b_{2}$ and $\Sigma b_{1} \wedge \Sigma b_{3}$. Again, and for similar reasons, one has a dichotomy between the finite and the infinite unitary groups, given as Theorems 1.1 and 1.2 of [3].

THEOREM 2.2. (a) The space $S X_{2}(n)$ is stably indecomposable (at the prime 2).

(b) The spectrum $S X_{2}(\infty)$ splits as a wedge of two indecomposable pieces if the prime 3 is inverted.

The techniques discussed here for the unitary group are not appropriate for the real orthogonal group. In the symplectic case, the second summand of the "Miller splitting" as in Theorem 2.2 of $S p(n)$ is indecomposable in the finite and infinite cases. But the situation for $\Omega S p(n)$ is quite different $[8,6,5]$.

\section{References}

[1] A. Baker, F. Clarke, N. Ray and L. Schwartz, On the On the Kummer congruence and the stable homotopy of BU, Trans. Amer. Math. Soc. 316 (1989), 385-432.

[2] M. C. Crabb, On the stable splitting of $U(n)$ and $\Omega U(n)$, Algebraic Topology, Barcelona, 1986, L. N. M 1298, 35-53, Springer, Berlin, 1987.

[3] M. C. Crabb and J. R. Hubbuck, The stable decomposition of $U(n)$, to appear in Proc. A. Roy. Soc. Edin. 127 (1997).

[4] M. C. Crabb and J. R. Hubbuck, The stable decomposition of $\Omega S U(n)$, manuscript, 1997.

[5] M. C. Crabb and S. A. Mitchell, The loops on $U(n) / O(n)$ and $U(2 n) / S p(n)$, Math. Proc. Cambridge Phil. Soc. 104 (1988), 95-103.

[6] J. R. Hubbuck, Some stably indecomposable loop spaces, Homotopy Theory and Related Topics, Kinosaki 1988, L. N. M. 1418, 70-77, Springer, Berlin (1990).

[7] J. R. Hubbuck, Numerical Forms, J. Lond. Math. Soc. 55 (1997), 65-75.

[8] M. J. Hopkins, Stable decompositions of certain loop spaces, Ph.D. thesis, North Western Univ., 1984.

[9] H. R. Miller, Stable Splitting of Stiefel Manifolds, Topology 24 (1988), 411-419.

[10] V. P. Snaith, Algebraic K-theory and localised stable homotopy theory, Mem. Amer. Math. Soc. 43 (1975), 235-255. 